\title{
Guidelines for Reporting Health Research: A User's Manual
}

\author{
David Moher, Douglas G Altman, Kenneth F Schulz, Iveta Simera, Elizabeth Wager \\ (Editors). John Wiley \& Sons, 2014, \$54.95, paperback, 344 pages. \\ ISBN 978-0-470-67044-6
}

\author{
Philip M. Jones, MD (D)
}

Received: 28 June 2015/Accepted: 13 July 2015/Published online: 4 August 2015

(C) Canadian Anesthesiologists' Society 2015

Whether they are conducting randomized clinical trials (RCTs), retrospective or prospective observational studies, or detailed genetic analyses, ultimately, the time will come for all researchers to prepare a write-up of their results for presentation to the global community. Just as there are best practices in designing and conducting clinical research, there are also best practices in reporting clinical research. Nevertheless, given the sheer number of study designs and the peculiarities of reporting each design, it can be difficult for researchers and those adjudicating research, such as peer reviewers, journal editors, and publishers, to remember all the optimal reporting practices.

In this regard, Guidelines for Reporting Health Research: A User's Manual is a useful reference book for clinical researchers, peer reviewers, journal editors, and publishers. Written by the original guideline authors, the book essentially presents and summarizes the various research reporting guidelines developed over the years in an effort to promote best practices in research reporting. Most Journal readers will already be familiar with one such guideline, the Consolidated Standards of Reporting Trials (best known by its acronym-CONSORT) statement, which has dramatically improved the reporting quality of RCTs over roughly the past 15 years. Nevertheless, many more such guidelines exist for various study designs: STROBE (for observational studies), STARD (for diagnostic accuracy studies), SURGE (for reports of surveys), and PRISMA (for

P. M. Jones, MD ( $\square)$

Departments of Anesthesia \& Perioperative Medicine and

Epidemiology \& Biostatistics, University of Western Ontario,

London, ON, Canada

e-mail: pjones8@uwo.ca reporting systematic reviews and meta-analyses). In addition, there is a whole series of extensions to the central CONSORT guidelines for different RCT designs, such as non-inferiority trials, equivalence trials, pragmatic trials, and cluster randomized trials. With all these guidelines, it would be most convenient to have a central resource with access to all of the reporting guidelines, and that's where this book shines.

The book begins with a few introductory chapters on why open and transparent reporting of medical research is a good thing, how the first reporting guidelines were developed, and how reporting guidelines are best incorporated into your workflow. The opening chapters also include an introduction to the EQUATOR (Enhancing the Quality and Transparency of Health Research) network which incorporates all of the various reporting guidelines on its website. ${ }^{\mathrm{A}} \mathrm{A}$ separate chapter is devoted to each of the distinct study designs, and there is consistency in the layout of each chapter: the same headings describe when and how to use the guideline, how the guideline was developed, whether there is any evidence on the effectiveness of the guideline, limitations to the guideline, and future plans to update the guideline. One of my favourite sections in each chapter is the Creators' preferred bits whereby the authors elaborate either on aspects of the guideline that they find particularly important or on certain areas where they have observed authors commonly make mistakes. Finally, each chapter also includes the checklist for the guideline being discussed. Having this book thus amounts to having "onestop shopping" for all the current reporting guidelines at your fingertips.

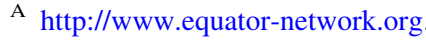


Beyond the chapters that summarize the various reporting guidelines, useful chapters at the end of the book focus on the correct ways to present the results of statistical analyses, how best to present data in figures and tables, and how best to present clinical and laboratory images in publications. There are some very good examples of figures that have high data density yet are easy to understand; a particularly good example is the figure on the diagnostic accuracy of ultrasonography in the emergency department. These chapters are useful for all researchers. Nevertheless, since tables of results are often poorly presented in study reports, it would have been useful to include a typical results table for an RCT (with comments on how best to present categorical variables, normally distributed continuous variables, and nonnormally distributed continuous variables) as well as a typical results table for an observational study (with comments on how best to present the results of univariable and multivariable regression analysis).
Although this book is suitable for all researchers, it will have the most impact on researchers who use a variety of study designs in their investigations. For instance, if researchers perform only RCTs, then it is probably easier to download one or two of the CONSORT guidelines. Similarly, if they perform only systematic reviews, the PRISMA guidelines will suffice. Then again, if they sometimes perform RCTs but also participate in observational studies, systematic reviews, and surveys, then it may be more convenient to use this book and have ease of access to all guidelines in one volume. Journal editors, editorial board members, and publishers would all benefit from keeping a copy of this book on hand. Finally, trainees interested in clinical research would benefit from reading this book from cover to cover as it will help them acquire a firm understanding of the best reporting practices for most types of clinical research.

Conflicts of interest None declared. 